\title{
Early Assessment of Left Ventricular Function by Layer-Specific Strain and Its Relationship to Pulsatile Arterial Load in Patients with Coronary Slow Flow
}

\author{
Jing Shi, ${ }^{1 *}$ MD, Yumeng Xing, ${ }^{1 *}$ MD, Juying Qian, ${ }^{2}$ MD, Hongbo Yang, ${ }^{2}$ MD, Yan Yan, ${ }^{2}$ MD, \\ Qing Li, ${ }^{2} \mathrm{MD}$, Limin Luo, ${ }^{3} \mathrm{MD}$, Dehong Kong, ${ }^{1} \mathrm{MD}$, Cuizhen Pan, ${ }^{1} \mathrm{MD}$ and Xianhong Shu, ${ }^{1} \mathrm{MD}$
}

\begin{abstract}
Summary
Previous studies reported a controversial left ventricular (LV) function impairment and pathophysiology in patients with coronary slow flow (CSF). Greater arterial load has been shown to increase aortic impedance and endothelial shear stress, potentially affecting coronary anatomy and function. We investigated LV systolic function by a new layer-specific strain technology and assessed the association between pulsatile arterial load and contractility.

A total of 70 patients with CSF and 50 controls with normal coronary angiography were included in the study. Layer-specific longitudinal and circumferential strains were assessed from endocardium, midmyocardium, and epicardium (global longitudinal strain (GLS)-endo, GLS-mid, GLS-epi and GCS-endo, GCSmid, GCS-epi) by two-dimensional speckle tracking imaging (2D-STI). Pulsatile arterial load was estimated by indexed arterial compliance (ACI). Layer-specific GLS showed a decreasing gradient from the endocardium to the epicardium in both the controls and CSF group. GLS-endo and GLS-mid in the CSF group were significantly lower than the control group (all $P<0.05$ ). Layer-specific longitudinal strain showed a good correlation with the number of affected coronary arteries (all $P<0.05$ ) and the mean thrombolysis in the myocardial infarction frame count (TFC) (all $P<0.05)$. ACI was lower in patients with CSF $(P=0.005)$, and ACI was correlated negatively with layer-specific GLS (all $P<0.05$ ).

Layer-specific evaluation of the LV provides an understanding of the layer-specific properties of the LV wall and the possible process of the LV impairment in patients with CSF. Greater pulsatile arterial load, as manifested by a lower ACI, is coupled with worse LV longitudinal function in patients with CSF.
\end{abstract}

(Int Heart J 2019; 60: 586-592)

Key words: Two-dimensional, Speckle-tracking, Myocardial ischemia, Arterial compliance

$\mathrm{C}$ oronary slow flow (CSF) is characterized by delayed opacification of coronary arteries in the presence of normal coronary angiogram. ${ }^{1)}$ The exact pathophysiologic mechanisms of CSF have not been established. Endothelial dysfunction, microvascular dysfunction, and occlusive disease of the small coronary arteries were reported to be associated with $\mathrm{CSF}^{2,3)}$ Lower arterial compliance has been shown to increase aortic impedance and endothelial shear stress, potentially affecting coronary anatomy and function. ${ }^{4.5)}$ Previous studies reported controversial left ventricular (LV) function impairment in patients with CSF. In addition, most of these studies merely analyzed the LVcontractility without considering the distinction between different layers of the myocardium and pulsatile arterial load condition. ${ }^{6.7)}$ The
LV wall of the heart comprises an endocardial, a midmyocardial, and an epicardial layer. ${ }^{8}$ Tissue doppler imaging and magnetic resonance imaging have been used to obtain insight into layer-specific differences in myocardial function. ${ }^{9-12)}$ However, most of these techniques are not widely available and their application is time consuming.

\section{Editorial p.495}

Recent two-dimensional speckle tracking imaging (2 D-STI) software allows separate evaluation of endocardial, mid-cardial, and epicardial myocardial deformation. Therefore, we conducted this study to evaluate the effects of CSF on LV myocardial contractility of each myocardial layer and to assess the relationship between the layer-

From the ${ }^{1}$ Department of Echocardiography, Zhongshan Hospital of Fudan University, Shanghai Institute of Medical Imaging, Shanghai Institute of Cardiovascular Diseases, Shanghai, China, ${ }^{2}$ Department of Cardiology, Zhongshan Hospital of Fudan University, Shanghai Institute of Cardiovascular Diseases, Shanghai, China and ${ }^{3}$ Department of Echocardiography, Xiamen Branch, Zhongshan Hospital of Fudan University, Xiamen, China.

*These authors contributed equally to this work.

This study was supported by grants 17ZR1427200 and 18ZR1436100 from the Shanghai Science and Technology Commission, grant 20164 Y0076 from the Shanghai Health and Family Planning Commission, and grant 81701694 from the National Natural Science Foundation of China.

Address for correspondence: Cuizhen Pan, MD, Department of Echocardiography, Zhongshan Hospital of Fudan University, 180 Fenglin Road, Shanghai 200032, China. E-mail: pan.cuizhen@zs-hospital.sh.cn

Received for publication August 17, 2018. Revised and accepted November 7, 2018

Released in advance online on J-STAGE April 25, 2019.

doi: $10.1536 /$ ihj. $18-495$

All rights reserved by the International Heart Journal Association. 
specific strain and the pulsatile arterial load in patients with CSF

\section{Methods}

Study population: Based on the recommendations of Gibson, et al. $^{13)}$ a total of 70 patients with slow coronary flow and otherwise normal coronary arteries (CSF group) and 50 age and sex-matched patients with normal coronary angiograms(control group) were prospectively recruited from 4,015 consecutive patients with coronary risk factors who received their first diagnostic coronary angiography for evaluation of angina in our center between October 2014 and May 2017. Patients with known coronary artery disease, visualized coronary plaque in coronary angiography, left ventricular systolic dysfunction (ejection fraction $<55 \%$ ), valvular heart disease, cardiac rhythm other than sinus, malignancy, chest pain longer than 6 months, and renal and hepatic insufficiency were excluded from the study. The institutional ethics review board of the hospital approved the study.

TFC and the definition of CSF: A Coronary arteriography was performed by a radial approach using the $5 \mathrm{~F}$ TIG catheters (TEURMO, Japan) and iopamiro (Bracco Sine Pharmaceutical Corp., Shanghai, China) as a contrast agent. Cine frames were counted according to the criteria for each territory defined by Gibson, et al. For the quantitative measurement of coronary blood flow, the time elapsed from the appearance of the contrast agent until it reached the distal end of either the left anterior descending artery (LAD), the circumflex artery $(\mathrm{Cx})$, or the right coronary artery (RCA) in terms of cine-frame count was considered to be the thrombolysis in the myocardial infarction frame count (TFC). The first frame used for TFC is the first frame in which dye fully enters the artery. This occurs when the following three criteria are met: (1) A column of nearly full or fully concentrated dye must extend across the entire width of the origin of the artery; (2) Dye must touch both borders of the origin of the artery; and (3) There must be an antegrade motion to the dye. The last frame is counted or included as one of the frames and is defined as the frame when dye first enters the distal landmark branch. The following distal landmark branches are used for analysis: the distal bifurcation of the LAD (i. e, the "mustache," "pitchfork," or "whale's tail"); in the circumflex system, the distal bifurcation of the segment with the longest total distance; and in the RCA, the first branch of the posterolateral artery. The distal end was defined as the distal bifurcation for LAD and $\mathrm{Cx}$ and first branch of the posterolateral artery for RCA. Then, the final count was subtracted from the initial count, and the exact TFC was calculated for the given artery. However, it was divided by 1.7 when LAD was the case for adjusted correction. The cut-off values were defined according to the TIMI frame count method by Gibson, et al. $(36 \pm 2.6$ for $\mathrm{LAD}, 22.2 \pm 4.1$ for $\mathrm{Cx}$, and $20.4 \pm 3.0$ for RCA). ${ }^{13)}$ Two separate cardiologists undertook the TFC with a third cardiologist as adjudicator in cases of dispute.

2Dechocardiography: Echocardiography was performed with the subjects at rest in the left lateral decubitus position with a commercially available system (M3S probe,
Vivid E9, GE Medical systems, Oslo, Norway) soon after patients were discharged from the catheter laboratory. Routine grayscale 2D cine loops from 3 consecutive beats were obtained at end-expiratory apnea from standard 3 apical views (4-chamber, 2-chamber, and long-axis) and 3 short-axis views. Frame rates were $74.2 \pm 5 \mathrm{~Hz}$ for grayscale imaging. LV volume and ejection fraction were assessed by the biplane Simpson method using autoEF tracing of digital images. LV mass index, LAVI (left atrial volume index), mitrial flow E/A ratio, myocardial annular velocities, and E/e' were measured in accordance with guidelines. ${ }^{14)}$

2D-STI: All images were digitally stored on hard disks for offline analysis (EchoPAC version 201.x.x, GEVingmed, Horten, Norway). Grayscale images were analyzed in a 18-segmental model, according to the American Society of Echocardiography. ${ }^{15)}$ Myocardial function by strain was evaluated on a frame-by-frame basis by automatic tracking of acoustic markers (speckles) throughout the cardiac cycle (Figure1). The endocardial borders were traced in the end-systolic frame of the 2D images from the 3 apical views for analysis of longitudinal endocardial, mid-myocardial, and epicardial strains. Analysis of layerspecific circumferential strains were obtained from the parasternal short-axis view. All segmental values were averaged to the global longitudinal strain (GLS) and global circumferential strain (GCS) for each myocardial layer. Segments that failed to track properly were manually adjusted by the operator. Any segments that subsequently failed to track were excluded.

Assessment of pulsatile arterial load: Blood pressure was measured on the day of the echocardiography study, after withholding antianginal drugs and before vasodilator administration. Arterial load is separated into steady (systemic vascular resistance) and pulsatile (arterial compliance) components. Systemic vascular resistance was estimated as (MAP*80)/cardiac output. The stroke volume and PP ratio correlates well with the invasively measured arterial compliance ${ }^{16)}$ and is independently associated with $\mathrm{CV}$ events and mortality ${ }^{17}{ }^{17}$ thus, it was used as a measure of arterial compliance. We indexed arterial load to the body surface area (BSA) by dividing arterial compliance by the BSA (indexed arterial compliance, ACI) and multiplying the systemic vascular resistance by the BSA (indexed systemic vascular resistance, SVRI).

Measurement variability: Using the methods described above, 20 randomly chosen $2 \mathrm{D}$ echocardiographic images were analyzed by two observers and by one observer with a minimum time interval of 2 weeks for the measurement of GLS-endo, GLS-mid, GLS-epi (global longitudinal strain of the endocardial layer, global longitudinal strain of the mid-myocardial layer, and global longitudinal strain of the epicardial layer), GCS-endo, GCS-mid, and GCSepi (global circumferential strain of the endocardial layer, global circumferential strain of the mid-myocardial layer, and global circumferential strain of the epicardial layer). The measurements were performed on the same cardiac cycle of the same image. All readers were blinded to previous measurements. Interobserver and intraobserver variability was assessed using the coefficient of variation (the absolute difference between repeated measurements as a 

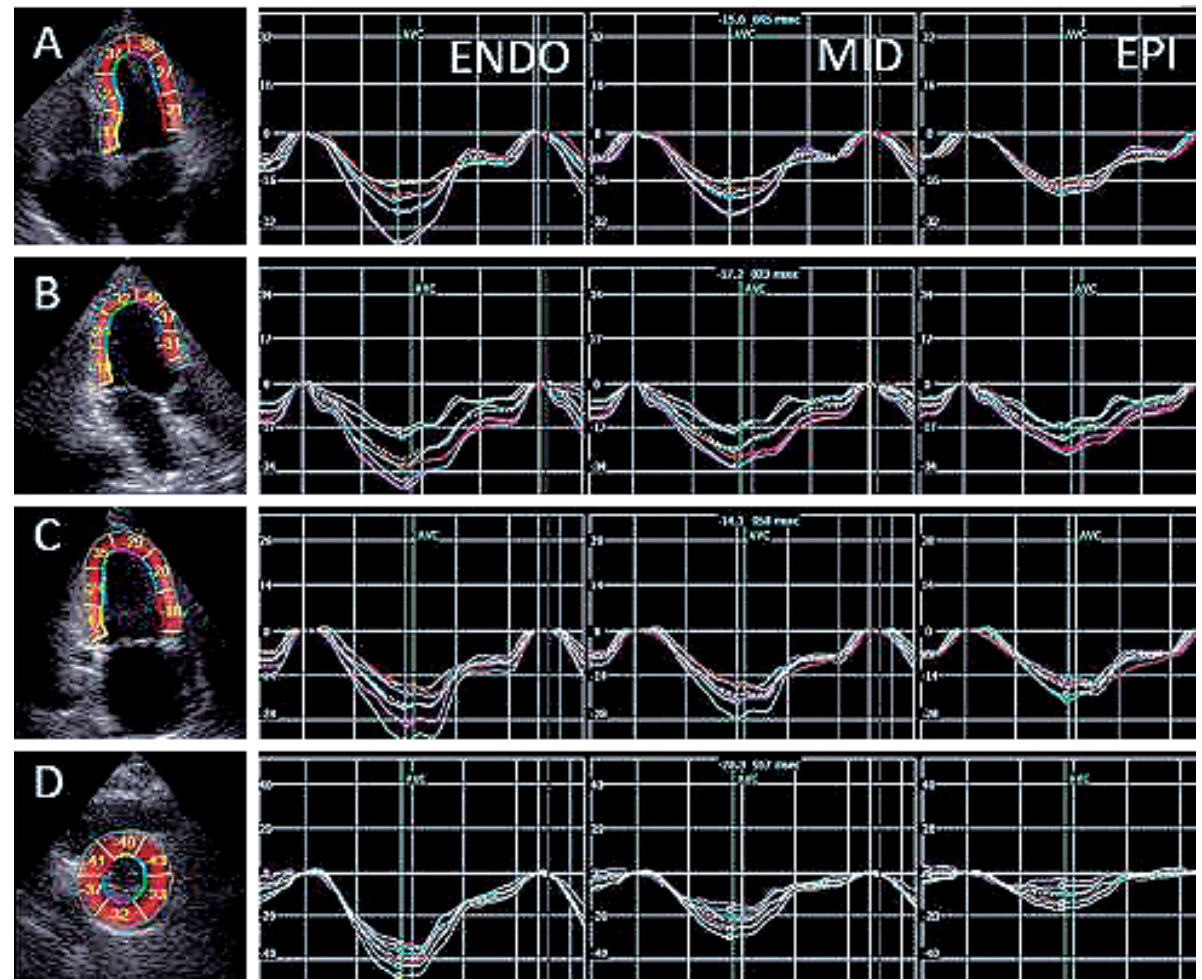

Figure 1. Three-layer longitudinal strain (apical view) and circumferential strain (short-axis view) in a control subject. From top to bottom; A: Apical 4-chamber view; B: Apical long-axis view, C: Apical 2-chamber view; D: Short-axis view at the level of the mitral valve. An endocardial-to-epicardial gradient (endocardial strain is highest and epicardial strain is lowest) of longitudinal and circumferential strain existed in control subjects. ENDO indicates endocardial layer; MID, mid-myocardial layer; and EPI, epicardial layer.

percentage of their mean value) and Bland-Altman analysis.

Statistical analysis: Continuous variables were presented as the mean \pm the standard deviation, unless otherwise stated. The prevalence of categorical variables was given. A student $t$-test and student $\chi^{2}$ tests were used for comparison of continuous and categorical variables, respectively. Correlations were evaluated using Spearman and Pearson correlation analyses where appropriate. All statistical analyses were performed using SPSS for Windows version19 (SPSS Inc, Chicago, IL). A 2-tailed P value of less than 0.05 was considered significant.

\section{Results}

Eight patients with CSF and two controls were excluded because of poor echocardiographic images and inadequate tracking quality of more than one segment in at least one apical view during the analysis of 2D-STI data. The presence of hypertension, diabetes, a smoking habit, a lipid profile, and fasting plasma glucose levels were similar in patients with CSF and the control group. Baseline characteristics of the study and the control group are presented in Table I.

The mean TFC was markedly high in the slow coronary flow group as compared to the control group (43.3 \pm 9.6 versus $15.1 \pm 2.7, P<0.001$, Table I). Of the 70 study patients, $68(97 \%)$ demonstrated slow coronary flow in the left anterior descending artery, $66(94 \%)$ in the circumflex artery, and $63(90 \%)$ in the RCA. Twenty-six patients had a slow coronary flow in three vessels and, five in two.

Two-dimensional echocardiographic data and global layer-specific strain were given in Table II. Left ventricular global systolic functions analyzed by the Simpson method, and the end-diastolic volume, end-systolic volume (ESV), LV wall thickness, LVMI, LAVI, E/A ratio, and E/e' ratio were not different in both groups. SV was significantly lower in the patients with CSF. Layerspecific GLS showed a decreasing gradient from the endocardium to the epicardium in both controls and the CSF group (Figures 1,2). GLS-endo and GLS-mid was significantly lower in the CSF group compared with the control group $(-23.0 \pm 3.3$ versus $-24.6 \pm 3.1,-20.0 \pm 2.8$ versus $-21.6 \pm 2.9$, all $P<0.05$ ). Patients with CSF showed generally lower three-layer global circumferential strain than the control group; however, the difference between these parameters generally did not reach statistical significance.

The relationships of layer-specific GLS measurements with the number of affected coronary arteries and TFC among patients with CSF are presented in Table III. Thelayer-specific GLS correlated negatively with the number of affected coronary arteries $(r=-0.44,-0.43,-0.35$, all $P<0.05)$ and with the TFC $(r=-0.31,-0.29,-0.45$, all $P<0.05)$ in patients with CSF.

SVRI was higher $(4978.6 \pm 1223.2$ versus $4223.7 \pm$ 
Table I. Basal Characteristics in the CSF and Control Group

\begin{tabular}{lcc}
\hline \multicolumn{1}{c}{ Variables } & CSF $(n=70)$ & Control $(n=50)$ \\
\hline Age $($ years $)$ & $56.9 \pm 7.8$ & $52.6 \pm 9.3$ \\
Female, $n(\%)$ & $30(43)$ & $23(46)$ \\
Heart rate $(\mathrm{bpm})$ & $72.1 \pm 8.3$ & $69.6 \pm 8.5$ \\
BMI $\left(\mathrm{kg} / \mathrm{m}^{2}\right)$ & $24.1 \pm 2.5$ & $23.9 \pm 3.1$ \\
DBP $(\mathrm{mmHg})$ & $120.9 \pm 7.8$ & $120.2 \pm 9.7$ \\
SBP $(\mathrm{mmHg})$ & $74.6 \pm 6.9$ & $76.2 \pm 5.4$ \\
Hypertension $(\%)$ & $22(31 \%)$ & $18(36 \%)$ \\
Diabetes mellitus $(\%)$ & $4(6 \%)$ & $2(4 \%)$ \\
Smoker, $n(\%)$ & $36(52)$ & $23(46)$ \\
TC $(\mathrm{mmol} / \mathrm{L})$ & $4.0 \pm 1.2$ & $3.9 \pm 1.2$ \\
TG $(\mathrm{mmol} / \mathrm{L})$ & $2.0 \pm 0.5$ & $5.2 \pm 0.5$ \\
FBG $(\mathrm{mmol} / \mathrm{L})$ & $5.3 \pm 0.3$ & $15.1 \pm 2.7$ \\
Mean TFC & $43.3 \pm 9.6^{\ddagger}$ & $4223.7 \pm 1200.3$ \\
SVRI $\left(\mathrm{dyne} \mathrm{seconds} / \mathrm{cm}^{5 * \mathrm{~m}^{2}}\right)$ & $4978.6 \pm 1223.2 *$ & $0.62 \pm 0.28$ \\
ACI $\left(\mathrm{mL} / \mathrm{mmHg} / \mathrm{m}^{2}\right)$ & $0.44 \pm 0.20^{\dagger}$ & \\
\hline
\end{tabular}

BMI indicates body mass index; SBP, systolic blood pressure; DBP, diastolic blood pressure; TC, total cholesterol; TG, triglyceride; FBG, fasting blood glucose; TFC, thrombolysis in myocardial infarction frame count; SVRI, indexed systemic vascular resistance; and ACI, indexed arterial compliance. ${ }^{*} P \leq 0.05$, ${ }^{\dagger} P \leq 0.01,{ }^{\ddagger} P<0.001$ for comparisons between CSF and control.

Table II. Two Dimensional Echocardiographic Data and Global Layer-Specific Strain in the CSF and Control Group

\begin{tabular}{lcc}
\hline & CSF $(n=31)$ & Control $(n=30)$ \\
\hline LVEF $(\%)$ & $62.1 \pm 4.3$ & $63.2 \pm 2.8$ \\
EDV $(\mathrm{mL})$ & $82.6 \pm 19.9$ & $79.6 \pm 18.0$ \\
ESV $(\mathrm{mL})$ & $32.4 \pm 9.3$ & $29.2 \pm 6.2$ \\
IVS $(\mathrm{mm})$ & $8.5 \pm 1.3$ & $8.5 \pm 1.2$ \\
PWT $(\mathrm{mm})$ & $8.1 \pm 1.1$ & $7.9 \pm 1.0$ \\
SV $(\mathrm{mL})$ & $49.2 \pm 10.4 *$ & $51.4 \pm 12.8$ \\
LVMI $\left(\mathrm{g} / \mathrm{m}^{2}\right)$ & $77.2 \pm 20.8$ & $75.1 \pm 25.6$ \\
LAVI $\left(\mathrm{cm}^{3} / \mathrm{m}^{2}\right)$ & $26.5 \pm 7.5$ & $26.8 \pm 7.0$ \\
Mitral E/A ratio & $1.2 \pm 0.4$ & $1.1 \pm 0.5$ \\
Septal E/e'ratio & $7.6 \pm 1.8$ & $7.5 \pm 2.0$ \\
GLS-endo $(\%)$ & $-23.0 \pm 3.1 *$ & $-24.6 \pm 3.3$ \\
GLS-mid $(\%)$ & $-20.0 \pm 2.8$ & $-21.6 \pm 2.9$ \\
GLS-epi $(\%)$ & $-18.9 \pm 2.8$ & $-19.1 \pm 2.5$ \\
GCS-endo $(\%)$ & $-34.2 \pm 6.6$ & $-36.5 \pm 4.6$ \\
GCS-mid $(\%)$ & $-24.3 \pm 2.9$ & $-25.4 \pm 3.0$ \\
GCS-epi $(\%)$ & $-17.2 \pm 3.7$ & $-18.1 \pm 3.6$ \\
\hline
\end{tabular}

LVEF indicates left ventricular ejection fraction; EDV, enddiastolic volume; ESV, end-systolic volume; IVS, interventricular septum; PWT, posterior wall thickness; SV, stroke volume; LVMI, left ventricular mass index; LAVI, left atrial volume index; GLS-endo, global longitudinal endocardial strain; GLS-mid, global longitudinal mid-myocardial strain; GLS-epi, global longitudinal epicardial strain; GCS-endo, global circumferential endocardial strain; GCS-mid, global circumferential mid-myocardial strain; and GCS-epi, global circumferential epicardial strain. ${ }^{*} P \leq 0.05,{ }^{\dagger} P \leq 0.01$ for comparisons between CSF and control.

1200.3, $P=0.012)$ and ACI was lower $(0.44 \pm 0.20$ versus $0.62 \pm 0.28, P=0.005)$ in patients with CSF, confirming greater steady and pulsatile arterial load in CSF patients (Table I). No correlation was observed between the layer-specific GLS and SVRI. ACI was correlated negatively with the layer-specific GLS $(r=-0.42,-0.44$, -0.55 , all $P<0.05$ ) (Table III).

Observer variability: Intraobserver coefficients of vari- ation for GLS-endo, GLS-mid, GLS-epi, GCS-endo, GCSmid, and GCS-epi were $5.4 \pm 4.5 \%, 5.4 \pm 4.6 \%, 6.2 \pm$ $4.3 \%, 6.2 \pm 3.7 \%, 9.0 \pm 1.0 \%$, and $8.6 \pm 5.0 \%$, respectively. Corresponding interobserver coefficients of variation were $5.2 \pm 3.8 \%, 5.7 \pm 4.0 \%, 3.2 \pm 3.6 \%, 6.0 \pm$ $4.0 \%, 8.6 \pm 7.6 \%$, and $11.2 \pm 7.7 \%$, respectively. The results concerning interobserver and intraobserver agreement for layer-specific myocardial strain parameters assessed by Bland-Altman analysis are displayed in Figure 3.

\section{Discussion}

This is the first study, to our knowledge, to assess layer-specific GLS and the association with a pulsatile arterial load in new-diagnosed patients with CSF. The main findings of this study are the following: (1) patients with CSF had worse function in all three myocardial layers assessed by GLS compared with control subjects. GLS-endo and, GLS-mid in the CSF group were significantly lower than the control group $(P=0.026,0.027)$, (2) layerspecific GLSin the CSF group was related to the number of the affected coronary arteries and their TFC, and (3) SVRI was higher and ACI was lower in patients with CSF. ACI was correlated negatively with layer-specific GLS.

The coronary slow flow phenomenon is an angiographic observation characterized by angiographically normal or near-normal epicardial coronary arteries with delayed dye opacification of the distal vasculature. ${ }^{1)}$ Previous studies suggested that a combination of structure and functional obstruction exists in the coronary microcirculation in patients with CSF. ${ }^{1,18-21)}$ The presence of myocardial ischemia in patients with CSF has been difficult to demonstrate consistently both by clinical and echocardiographic markers. A possible explanation for this observation would include that the techniques used to detect myocardial ischemia may be relatively insensitive for the microvascular disorder. During systole, the degree of con- 

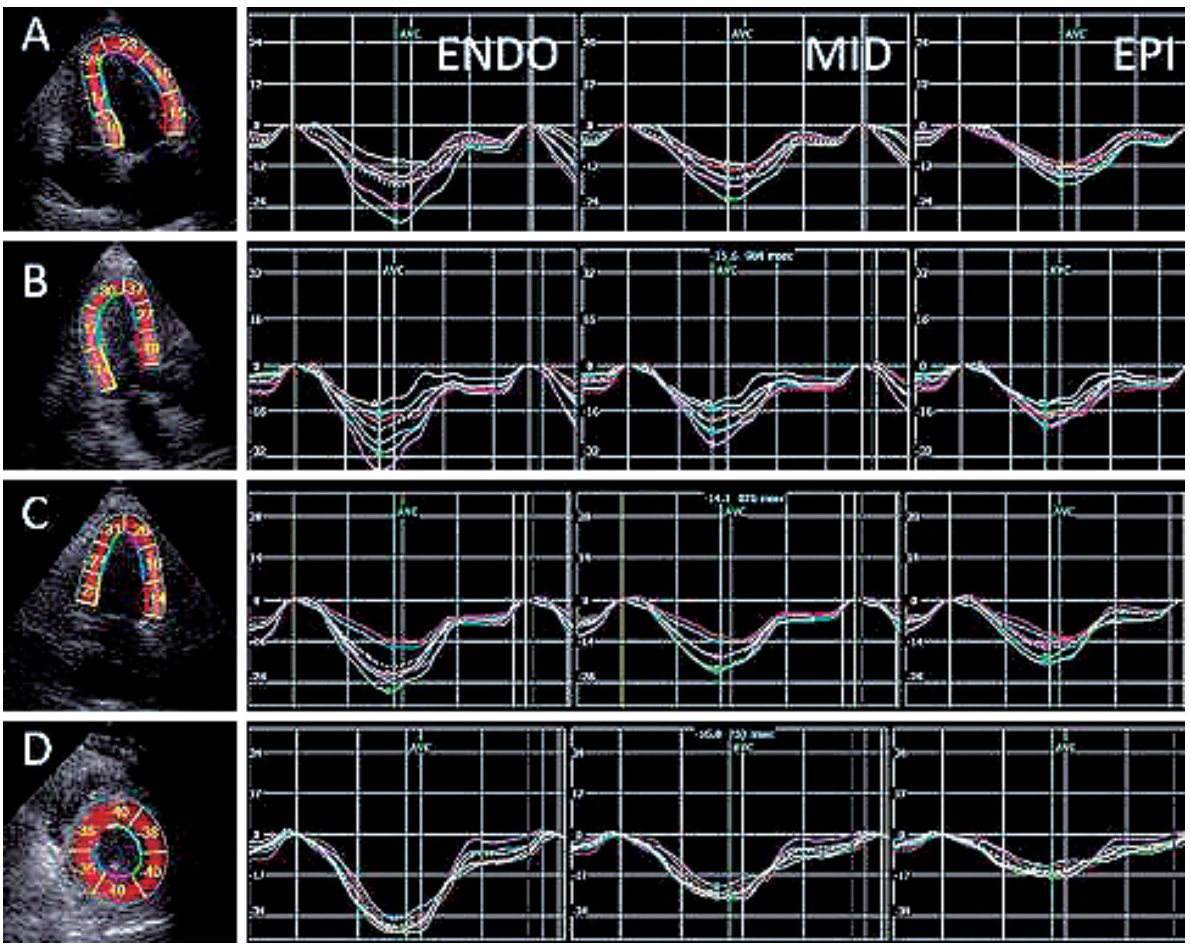

Figure 2. Three-layer longitudinal strain (apical view) and circumferential strain (short-axis view) in a patient with CSF. From top to bottom; A: Apical 4-chamber view; B: Apical long-axis view, C: Apical 2-chamber view; D: Short-axis view at the level of the mitral valve. An endocardial-to-epicardial gradient of longitudinal and circumferential strain still existed in patients with CSF. Compared with control subjects, the amplitude of these strain curves was reduced in most of the segments. ENDO indicates endocardial layer; MID, mid-myocardial layer; and EPI, epicardial layer.

Table III. Correlation Analysis of Layer-Specific Longitudinal Strain in Patients with CSF

\begin{tabular}{llcc}
\hline & $\begin{array}{c}\text { Mean TFC } \\
r(P \text { value })\end{array}$ & $\begin{array}{c}\text { Number of } \\
\text { affected arteries } \\
r(P \text { value })\end{array}$ & $\begin{array}{c}\text { ACI } \\
r(P \text { value })\end{array}$ \\
\hline GLS-endo (\%) & $-0.31(0.01)$ & $-0.44(0.016)$ & $-0.42(0.005)$ \\
GLS-mid (\%) & $-0.29(0.043)$ & $-0.43(0.021)$ & $-0.44(0.025)$ \\
GLS-epi (\%) & $-0.45(0.032)$ & $-0.35(0.045)$ & $-0.55(0.032)$ \\
\hline
\end{tabular}

TFC indicates thrombolysis in myocardial infarction frame count; ACI, indexed arterial compliance; GLS-endo, global longitudinal endocardial strain; GLS-mid, global longitudinal mid-myocardial strain; and GLS-epi, global longitudinal epicardial strain.

tribution of LV contraction is different in each layer. The endomyocardial layer is thickened predominantly during systole, and a decreasing functional gradient from the endocardium to the epicardium is observed. ${ }^{22-24)}$ Previous studies have reported layer-specific strain differences in patients with a non-ST-segment elevation acute coronary syndrome and chronic ischemic cardiomyopathy. ${ }^{25,26)}$ Wang, et $a l .{ }^{27)}$ have analyzed the LV three-layer strain in patients with CSF; however, they did not show the layerspecific difference in the strain values reflecting the inhomogeneous pathologic change in the endocardium and the midwall layer, which was well evaluated in our study.

2D-STI has been performed extensively to evaluate LV function in various cardiac conditions. LV strain analysis objectively quantified myocardial function and successfully evaluated the subclinical changes in patients with CSF. Recently, evaluating layer-specific strain of the LV myocardium with LV strain analysis has also become possible, which enables more precise evaluation of myocardial mechanics. ${ }^{26,28)}$

In the present study, layer-specific GLS using 2DSTI was successfully obtained, and it properly evaluated LV function in patients with CSF. The value of layerspecific GLS in the control groupwas similar to our previous report. ${ }^{29)}$ The decreased layer-specific LS of the endocardium and midcardium in new-diagnosed patients with CSF indicated the possible process of LV functional impairment in patients with CSF, which begins from the endomyocardial layer of the LV. Our study also showed that the mean TFC and the number of affected coronary arteries were negatively correlated with the contractility of all myocardial layers which emphasizes the importance of close follow-ups of patients with CSF involving three coronary arteries. The layer-specific analysis of LV strain is expected to detect the functional impairment of the LV more sensitively at an earlier stage than it will detect the other parameters.

In our study, patients with CSF had a lower ACI and higher SVRI than the control group. In addition, we have demonstrated that the lower ACI, leading to a greater pulsatile arterial load, imposed upon the LV, is associated with decreased layer-specific GLS in patients with CSF. 

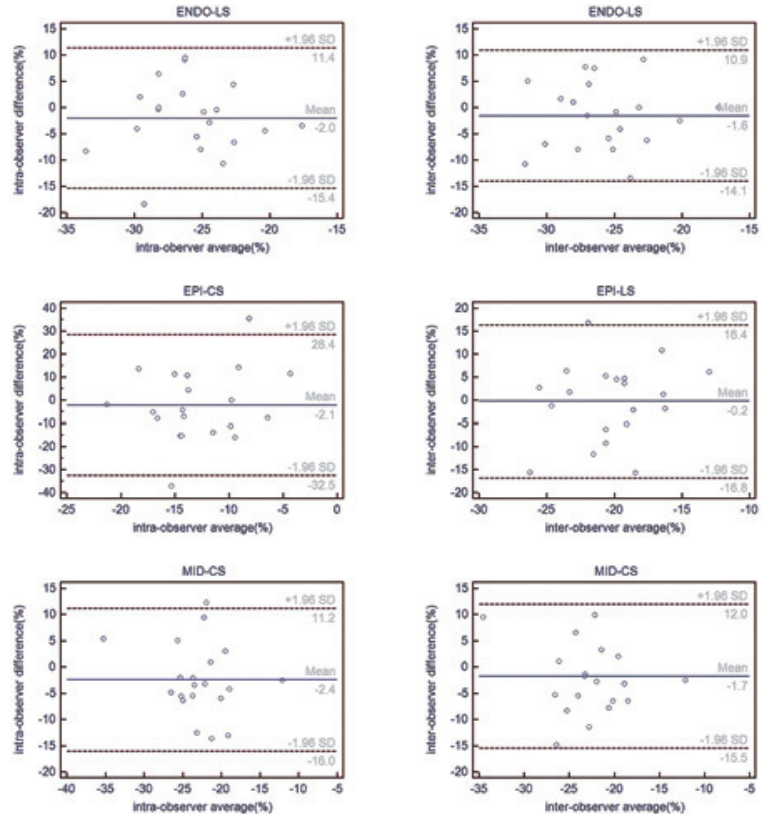
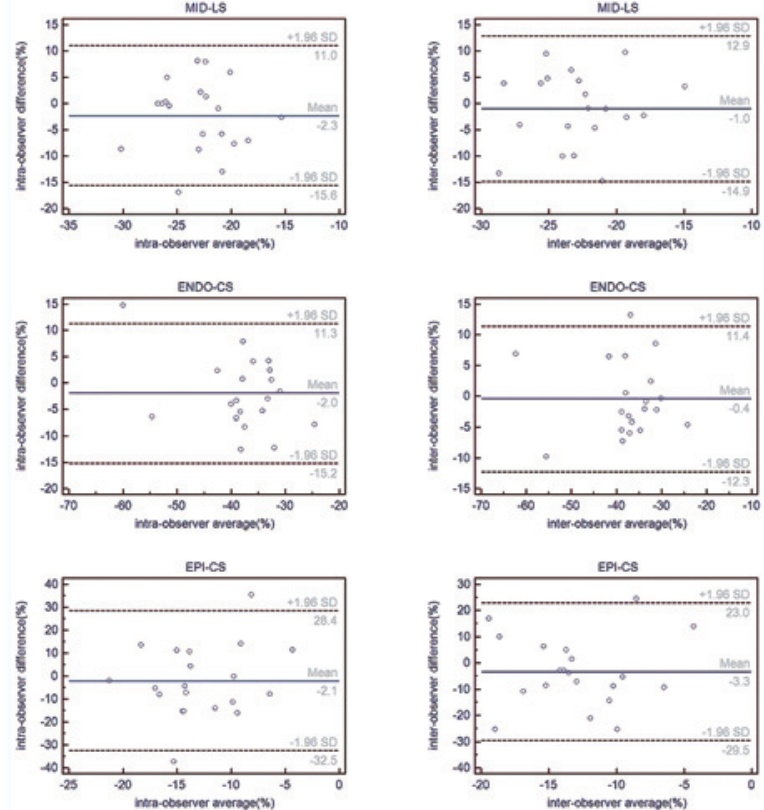

Figure 3. Bland-Altman plots showing interobserver and intraobserver differences and limits of agreement of layer-specific myocardial strain parameters.

Mechanisms through which low arterial compliance may adversely affect the coronary circulation include the fact that arterial compliance, increasing pressure pulsatililty, and decreasing diastolic pressure at the aortic root, may detrimentally affect coronary microvascular health. Alternatively, it is also possible that these changes occur in parallel, with systemic abnormalities associated with CSF simultaneously impaired LV function and aortic compliance. Our cross-sectional study cannot infer directionality of these associations, nor cause or effect. However, this study provides a new novel viewpoint on which to base future mechanics and prospective studies.

Limitations: This was a single-center and low sample size study. The samples of different affected vessels groups were not equal, raising the possibility that the degree of vessel involvement effect may have not been adequately detected. Secondly, we did not measure invasively arterial stiffness and load. However, the SV and PP ratio is a validated global measure of arterial compliance shown to correlate highly with invasive measures, used in largescale studies, and this ratio is an independent predictor of cardiovascular events. Thirdly, our study focused on longitudinal and circumferential layer-specific strain, but not radial strain. We chose not to analyze radial strain because current software has methodological limitations. Lastly, the cross-sectional nature of our study precludes inferences about causality and temporality of the associations.

\section{Conclusions}

LV myocardial contractility was lower in the patients with CSF, and all three myocardial layers were affected, particularly the endocardial and midcardial layer. The extent of functional decline in contractility of the three myocardial layers, was related to the number of affected coro- nary arteries and the mean TFC. Lower arterial compliance, leading to greater arterial stiffness and pulsatile arterial load, is associated with decreased longitudinal LV contractility in patients with CSF. Our findings motivate further prospective studies that aimed at targeting arterial compliance and LV dysfunction in the early detection and prevention of cardiovascular disease in patients with CSF.

\section{Disclosure}

Conflicts of interest: None.

\section{References}

1. Tambe AA, Demany MA, Zimmerman HA, Mascarenhas E. Angina pectoris and slow flow velocity of dye in coronary arteries--a new angiographic finding. Am Heart J 1972; 84: 6671.

2. Mangieri E, Macchiarelli G, Ciavolella M, et al. Slow coronary flow: clinical and histopathological features in patients with otherwise normal epicardial coronary arteries. Cathet Cardiovasc Diagn 1996; 37: 375-81.

3. Pekdemir $\mathrm{H}$, Polat $\mathrm{G}$, Cin VG, et al. Elevated plasma endothelin-1 levels in coronary sinus during rapid right atrial pacing in patients with slow coronary flow. Int J Cardiol 2004; 97: 35-41.

4. Beale AL, Meyer P, Marwick TH, Lam CSP, Kaye DM. Sex differences in cardiovascular pathophysiology: Why women are overrepresented in heart failure with preserved ejection fraction. Circulation 2018; 138: 198-205.

5. Patel MB, Bui LP, Kirkeeide RL, Gould KL. Imaging microvascular dysfunction and mechanisms for female-male differences in CAD. JACC Cardiovasc Imaging 2016; 9: 465-82.

6. Kemaloglu OT, Eren M, Atasoy I, Gurol T, Soylu O, Dagdeviren B. Are biventricular systolic functions impaired in patient with coronoray slow flow? A prospective study with three dimensional speckle tracking. Int J Cardiovasc Imaging 2017; 33: 
675-81.

7. Narimani S, Hosseinsabet A, Pourhosseini H. Effect of coronary slow flow on the longitudinal left ventricular function assessed by 2-dimensional speckle-tracking echocardiography. J Ultrasound Med 2016; 35: 723-9.

8. Duncker DJ, Traverse JH, Ishibashi Y, Bache RJ. Effect of NO on transmural distribution of blood flow in hypertrophied left ventricle during exercise. Am J Physiol 1999; 276: H1305-12.

9. Hashimoto I, Li X, Hejmadi BA, Jones M, Zetts AD, Sahn DJ. Myocardial strain rate is a superior method for evaluation of left ventricular subendocardial function compared with tissue Doppler imaging. J Am Coll Cardiol 2003; 42: 1574-83.

10. Delfino JG, Fornwalt BK, Eisner RL, Leon AR, Oshinski JN Determination of transmural, endocardial, and epicardial radia strain and strain rate from phase contrast MR velocity data. J Magn Reson Imaging 2008; 27: 522-8.

11. Funabashi N, Takaoka H, Ozawa K, et al. Quantitative differentiation of LV myocardium with and without layer-specific fibrosis using mri in hypertrophic cardiomyopathy and layer-specific strain TTE analysis. Int Heart J 2018; 59: 523-30.

12. Funabashi N, Takaoka H, Ozawa K, Kobayashi Y. Endocardial fibrotic lesions have a greater effect on peak longitudinal strain than epicardial fibrotic lesions in hypertrophic cardiomyopathy patients. Int Heart J 2018; 59: 347-53.

13. Gibson CM, Cannon CP, Daley WL, et al. TIMI frame count: a quantitative method of assessing coronary artery flow. Circulation 1996; 93: 879-88

14. Lang RM, Badano LP, Mor-Avi V, et al. Recommendations for cardiac chamber quantification by echocardiography in adults: an update from the American Society of Echocardiography and the European Association of Cardiovascular Imaging. Eur Heart J Cardiovasc Imaging 2015; 16: 233-70.

15. Bachner-Hinenzon N, Ertracht O, Leitman M, et al. Layerspecific strain analysis by speckle tracking echocardiography reveals differences in left ventricular function between rats and humans. Am J Physiol Heart Circ Physiol 2010; 299: H664-72.

16. Chemla D, Hebert JL, Coirault C, et al. Total arterial compliance estimated by stroke volume-to-aortic pulse pressure ratio in humans. Am J Physiol 1998; 274: H500-5.

17. Fagard RH, Pardaens K, Staessen JA, et al. The pulse pressureto-stroke index ratio predicts cardiovascular events and death in uncomplicated hypertension. J Am Coll Cardiol 2001; 38: 22731.

18. Mangieri E, Macchiarelli G, Ciavolella M, et al. Slow coronary flow: clinical and histopathological features in patients with otherwise normal epicardial coronary arteries. Cathet Cardiovasc Diagn 1996; 37: 375-81.

19. Beltrame JF, Limaye SB, Horowitz JD. The coronary slow flow phenomenon--a new coronary microvascular disorder. Cardiology 2002; 97: 197-202.

20. Mosseri M, Yarom R, Gotsman MS, Hasin Y. Histologic evidence for small-vessel coronary artery disease in patients with angina pectoris and patent large coronary arteries. Circulation 1986; 74: 964-72.

21. Beltrame JF, Limaye SB, Wuttke RD, Horowitz JD. Coronary hemodynamic and metabolic studies of the coronary slow flow phenomenon. Am Heart J 2003; 146: 84-90.

22. Adamu U, Schmitz F, Becker M, Kelm M, Hoffmann R. Advanced speckle tracking echocardiography allowing a threemyocardial layer-specific analysis of deformation parameters. Eur J Echocardiogr 2009; 10: 303-8.

23. Carasso S, Biaggi P, Rakowski H, et al. Velocity vector imaging: Standard tissue-tracking results acquired in normals--the VVI-STRAIN study. J Am Soc Echocardiogr 2012; 25: 543-52.

24. Myers JH, Stirling MC, Choy M, Buda AJ, Gallagher KP. Direct measurement of inner and outer wall thickening dynamics with epicardial echocardiography. Circulation 1986; 74: 164-72.

25. Hamada S, Schroeder J, Hoffmann R, et al. Prediction of outcomes in patients with chronic ischemic cardiomyopathy by layer-specific strain echocardiography: A proof of concept. J Am Soc Echocardiogr 2016; 29: 412-20.

26. Sarvari SI, Haugaa KH, Zahid W, et al. Layer-specific quantification of myocardial deformation by strain echocardiography may reveal significant $\mathrm{CAD}$ in patients with non-ST-segment elevation acute coronary syndrome. JACC Cardiovasc Imaging 2013; 6: 535-44.

27. Wang Y, Ma C, Zhang Y, et al. Layer-specific analysis of left ventricular myocardial contractility in patients with coronary slow-flow phenomenon. J Clin Ultrasound 2016; 44: 429-36.

28. Williams LK, Urbano-Moral JA, Rowin EJ, et al. Velocity vector imaging in the measurement of left ventricular myocardial mechanics on cardiac magnetic resonance imaging: correlations with echocardiographically derived strain values. J Am Soc Echocardiogr 2013; 26: 1153-62.

29. Shi J, Pan C, Kong D, Cheng L, Shu X. Left ventricular longitudinal and circumferential layer-specific myocardial strains and their determinants in healthy subjects. Echocardiography 2016; 33: $510-8$. 